\title{
Integrin $\beta 1$ orchestrates the abnormal cell-matrix attachment and invasive behaviour of E-cadherin dysfunctional cells
}

\author{
Joana Figueiredo ${ }^{1,2} \cdot$ Rui M. Ferreira ${ }^{1,2} \cdot$ Han Xu $^{3} \cdot$ Margarida Gonçalves $^{1,4,5} \cdot$ André Barros-Carvalho $^{1,4}$. \\ Janine Cravo ${ }^{1,2} \cdot$ André F. Maia $^{1,4} \cdot$ Patrícia Carneiro ${ }^{1,2}$. Céu Figueiredo ${ }^{1,2,6}$. Michael L. Smith ${ }^{3}$. \\ Dimitrije Stamenovic ${ }^{3,7} \cdot$ Eurico Morais-de-Sá ${ }^{1,4} \cdot$ Raquel Seruca $^{1,2,6}$
}

Received: 3 May 2021 / Accepted: 19 August 2021 / Published online: 5 September 2021

(c) The Author(s) 2021

\begin{abstract}
Background Tumour progression relies on the ability of cancer cells to penetrate and invade neighbouring tissues. E-cadherin loss is associated with increased cell invasion in gastric carcinoma, and germline mutations of the E-cadherin gene are causative of hereditary diffuse gastric cancer. Although E-cadherin dysfunction impacts cell-cell adhesion, cell dissemination also requires an imbalance of adhesion to the extracellular matrix (ECM).

Methods To identify ECM components and receptors relevant for adhesion of E-cadherin dysfunctional cells, we implemented a novel ECM microarray platform coupled with molecular interaction networks. The functional role of putative candidates was determined by combining micropattern traction microscopy, protein modulation and in vivo approaches, as well as transcriptomic data of 262 gastric carcinoma samples, retrieved from the cancer genome atlas (TCGA).

Results Here, we show that E-cadherin mutations induce an abnormal interplay of cells with specific components of the ECM, which encompasses increased traction forces and Integrin $\beta 1$ activation. Integrin $\beta 1$ synergizes with E-cadherin dysfunction, promoting cell scattering and invasion. The significance of the E-cadherin-Integrin $\beta 1$ crosstalk was validated in Drosophila models and found to be consistent with evidence from human gastric carcinomas, where increased tumour grade and poor survival are associated with low E-cadherin and high Integrin $\beta 1$ levels.

Conclusions Integrin $\beta 1$ is a key mediator of invasion in carcinomas with E-cadherin impairment and should be regarded as a biomarker of poor prognosis in gastric cancer.
\end{abstract}

Keywords E-cadherin $\cdot$ Hereditary diffuse gastric cancer $\cdot$ Extracellular matrix $\cdot$ Invasion $\cdot$ Integrin $\beta 1$

\section{Introduction}

E-cadherin is an essential molecule for epithelial homeostasis by regulating epithelial architecture and tissue integrity [1]. In cancer, genetic and epigenetic alterations in the

Raquel Seruca

rseruca@ipatimup.pt

http://www.i3s.up.pt

1 i3S-Instituto de Investigação e Inovação em Saúde, University of Porto, Rua Alfredo Allen 208, 4200-135 Porto, Portugal

2 Institute of Molecular Pathology and Immunology of the University of Porto (IPATIMUP), 4200-135 Porto, Portugal

3 Department of Biomedical Engineering, Boston University, Boston, MA 02215, USA
E-cadherin gene $(\mathrm{CDHl})$ or aberrant protein expression are frequent and result in loss of cell-cell adhesion, increased cell invasion and metastasis [2]. In the hereditary form of diffuse gastric cancer, known as hereditary diffuse gastric cancer syndrome (HDGC), inactivating germline alterations 
of the $C D H 1$ gene are causative events [3, 4]. HDGC is a highly penetrant cancer syndrome characterized by multiple foci of isolated malignant cells that invade widely through the gastric wall $[4,5]$. Precursor lesions of invasive gastric cancer have been identified in $\mathrm{CDH} 1$ mutation carriers as in situ signet ring cell carcinoma (SRCC) or pagetoid spread of signet ring cells below the preserved epithelium of glands $[6,7]$. These early gastric lesions are a hallmark of the disease and provide unique evidence that, in the initial steps of the neoplastic process, E-cadherin dysfunctional cells lack cellular cohesion but maintain a close contact with the basement membrane-a specialized extracellular matrix (ECM) that supports and fine-tunes cellular functions $[6,7]$.

Despite progress in the characterization of invasive gastric cancer cells, the mechanisms underlying their aggressive spreading remain largely unknown. We hypothesize that E-cadherin mutations associated with HDGC lead to aberrant integrin activation and signalling, initiating a specific mechano-transduction pathway at the early steps of invasion. In the last few years, data have emerged demonstrating a complex interplay between cell-cell junctions and cell-ECM adhesion $[8,9]$. While cadherins are central for cell-cell contacts, integrins are the main regulators of the cell-ECM crosstalk, working as mechanical transducers and as signalling molecules $[1,10,11]$. In homeostasis, the relationship between cadherins and integrins is tightly controlled, whereas in cancer this crosstalk is dysregulated and highly associated with invasion and metastasis [11-13].

In this study, we dissected the cascade of events occurring at the interface of E-cadherin dysfunctional cells with the ECM. Our strategy encompassed a high-content screening approach coupled with molecular interaction networks to identify ECM components and receptors relevant for adhesion of E-cadherin mutant cells. Functional significance of the findings was subsequently addressed through a set of in vitro and in vivo assays, as well as transcriptomic data of gastric carcinoma samples, retrieved from the cancer genome atlas (TCGA). Overall, our results demonstrate that E-cadherin loss modifies physical and biochemical features of the cell-matrix interaction, and pinpoint Integrin $\beta 1$ as the key player in cadherin-mediated invasion. This data highlights ECM-specific receptors, such as integrins, as novel biomarkers of poor prognosis in gastric cancer with E-cadherin impairment.

\section{Materials and methods}

\section{Plasmids}

E-cadherin variants A634V (c.1901C > T), R749W (c.2245C > T) and V832M (c.2494G > A) were induced by site-directed mutagenesis in the entry vector $\mathrm{CDH} 1 \mathrm{pENTR}$
221 (Clone ID: IOH46767, Invitrogen, Grand Island, NY, USA) and subcloned into the pEF6/Myc-His vector (Invitrogen) by LR recombination [14]. The corresponding empty vector (Mock) was constructed by restriction of the $\mathrm{CDH}$ 1pEF6/Myc-His with BsrGI (Biolabs, Ipswich, MA, USA).

\section{Cell culture and transfection}

AGS cell line (gastric adenocarcinoma, ATCC number CRL1739) was maintained and transfected as described in Supplementary Materials and Methods.

\section{ECM microarrays}

The cell-matrix adhesion profile of AGS cells transfected with the wild-type and the A634V, R749W and V832M E-cadherin variants was determined using a MicroMatrix $^{\mathrm{TM}} 36$ cell culture system (MicroStem). A suspension of $2.5 \times 10^{5}$ cells were seeded on the array slides. Slides were incubated at $37^{\circ} \mathrm{C}$ under $5 \% \mathrm{CO}_{2}$ humidified air for $48 \mathrm{~h}$ to allow cell attachment. Cells were washed in PBS and fixed in ice-cold methanol for $20 \mathrm{~min}$. Nuclear staining was achieved with a $1 \mu \mathrm{g} / \mathrm{ml}$ DAPI solution. Image acquisition was performed on an IN Cell Analyzer 2000 (GE Healthcare) with a Nikon 10x/0.45NA objective. Nuclei segmentation was achieved with ilastik [15], and CellProfiler [16] was subsequently used for image analysis and quantification. Similarity between cell-matrix attachment profiles was determined through Pearson's correlation distances. Significant differences in the number of wild-type and mutant adherent cells were evaluated using linear discriminant analysis effect size (LEfSe). ECM compositions with LDA $>4$ and $P<0.05$ were considered significantly enriched.

\section{ECM-integrin network analysis}

A consensus list of receptors and corresponding ECM ligands was retrieved from published records [17, 18]. Based upon this data, all possible physical interactions between integrins and ECM compositions assayed in the array were analysed. The network diagram was created using Cytoscape (version 3.1.0) [19]. Relative frequency of each specific integrin subunit ( $\beta$ and $\alpha$ ) was calculated considering the total number of interactions with ECM compositions that induced significant alterations in mutant attachment (estimated using LEfSe analysis).

\section{Micropattern traction microscopy}

Micropattern traction microscopy was performed as previously described [20]. A detailed description is provided at Supplementary Materials and Methods. 


\section{Western blotting}

Cell lysates were analysed as described by Figueiredo et al. [14].

\section{Matrigel invasion assays}

Cell invasive abilities were assessed using matrigel invasion inserts suitable for 24-well-plates (Corning BioCoat). Detailed protocol available at Supplementary Materials and Methods.

\section{Cell topology analysis}

Cellular distribution patterns were examined using fluorescence images of DAPI-stained cells. Image denoising and nuclei segmentation were performed in each image by applying the Otsu method and the Moore-Neighbor tracing algorithm, modified by Jacob's stopping criteria [21]. Nuclei geometric centres were computed and connected using the Delaunay triangulation algorithm. Triangles composing cellular networks were analysed for features such as area and edges length.

\section{Drosophila strains and genetic manipulations}

A transgenic Drosophila model based on the Gal4/UAS system was used to target gene expression in fly tissues. For specific expression in border cells, we generated flies containing UAS-hE-cad WT or UAS-hE-cad R749W together with UAS-mCherry, which were all expressed using GAL4 driven from a border cell-specific promoter (slbo-Gal4, BDSC \#58435). For the genetic interaction analysis in the adult eye, we established fly lines carrying simultaneously hE-cad and one RNAi targeting $\beta P S$ integrin (RNAi \#1 obtained from BDSC \#27735 and RNAi \#2 obtained from BDSC \#33642) or UAS-mCherry (BDSC \#35787), as control for titration of the number of UAS lines in the organism. These lines were then crossed with the GMR-Gal4 line and the appropriate progeny was selected. Eye phenotypes of at least 200 progeny flies (per condition) from three independent experiments were evaluated under a Leica compound microscope. Digital images were processed using Adobe Photoshop CS6. For further details, see Supplementary Materials and Methods.

\section{TCGA data analysis}

RNA-seq data of 291 samples were retrieved from the supplemental data of Bass A et al. [22]. The data represent a data freeze from February 2, 2014 and is available at https:// gdc.cancer.gov/about-data/publications/stad_2014. RNAseq results include 262 gastric cancer cases and 29 adjacent non-tumour tissues (details in Supplementary Table 1 and Supplementary Materials and Methods).

\section{Statistical analysis}

Data normality was verified with D'Agostino-Pearson omnibus test. Differences in normal distributed data were analyzed with unpaired Student's $t$ test or with one-way ANOVA, while differences in non-normal distributed data were evaluated by Kruskal-Wallis corrected with Dunn's test for multiple comparisons. Statistical data analysis was performed using the GraphPad Prism software (version 7.04 ), where $P \leq 0.05$ was considered significant.

\section{Results}

\section{E-cadherin mutant cells display altered cell-matrix attachment profiles}

A634V, R749W and V832M are three E-cadherin variants reported as causative in patients with HDGC [23-26]. To investigate the effects of variants associated with HDGC in cell-matrix interactions, we have used cells transfected with wild-type E-cadherin or the different variants in an array of 36 combinations of ECM proteins (Fig. 1A). The number of cells attached to each ECM spot was quantified as a direct measure of cell-matrix adhesive ability, maintaining cell maximal biological activity. In general terms, each E-cadherin mutant has a clearly distinct attachment profile (Fig. 1B). When compared with cells expressing wild-type E-cadherin, the extracellular mutant A634V shows increased adhesion for a panel of ECM combinations, which is not so striking in the juxtamembrane R749W. In contrast, the expression of the V832M intracellular variant leads to an overall trend of lower number of adherent cells.

Matrix similarity analysis, based on Pearson correlation distance, demonstrates that all variants generate a significant different pattern from that displayed by wild-type E-cadherin cells $(P<0.0001$ for all variants, Fig. $1 \mathrm{C}-\mathrm{E})$. The A634V mutant presents the most distinct matrix from that of the wild-type reference (mean distance of 0.5458 in A634V and 0.1029 in the WT), while the R749W yields the closest adhesive activity (0.2600).

\section{E-cadherin mutants have common preferences regarding ECM composition}

Our next aim was to determine which ECM compositions would be advantageous to E-cadherin dysfunctional cells. Although attachment ability is variable across the three E-cadherin mutants, we observed that a panel of ECM combinations induces a consistent increase in the number 
A

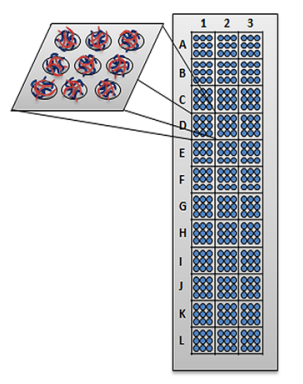

WT

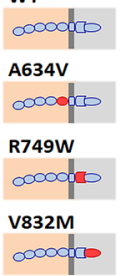

B

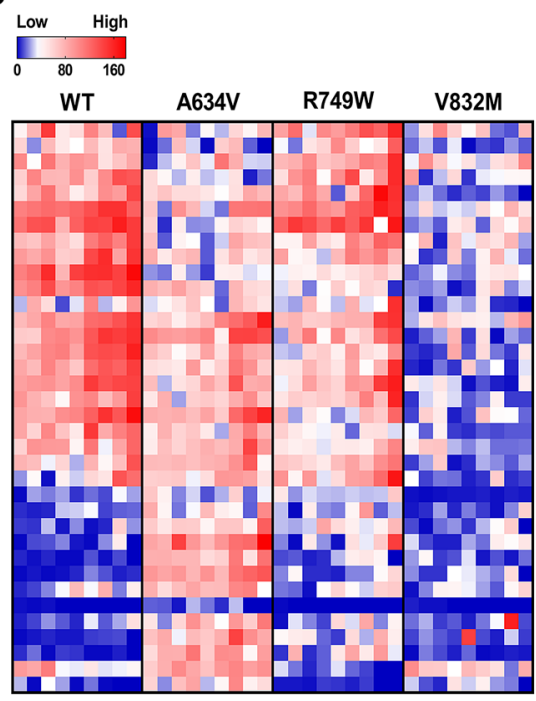

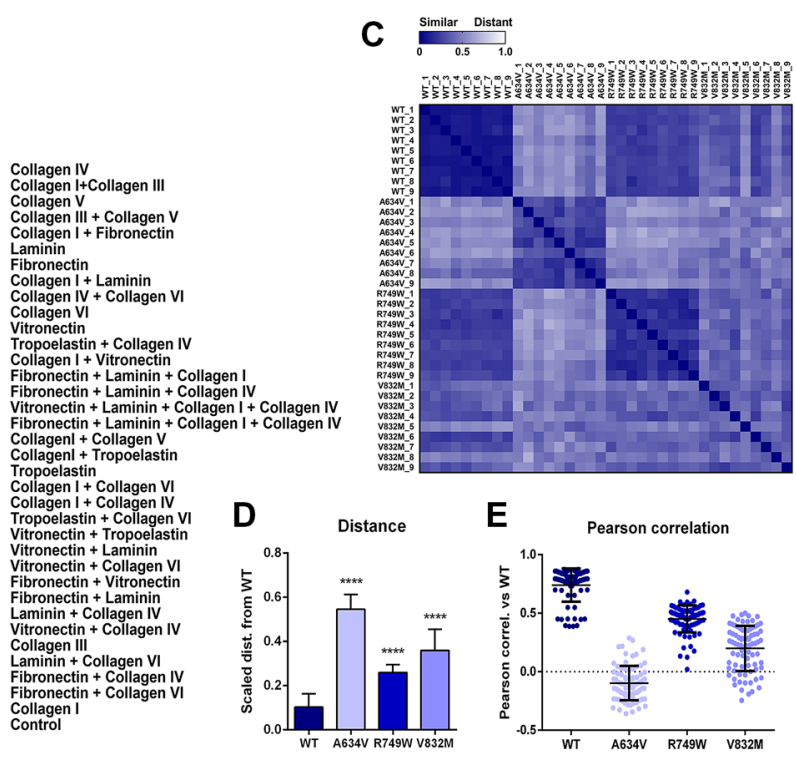

Fig. 1 Cell-matrix interaction induced by E-cadherin variants associated with HDGC. A An ECM array platform was used to evaluate the adhesion profile of human gastric cells transfected with wild-type E-cadherin or with cancer-associated mutants A634V, R749W and V832M that affect, respectively, the extracellular, the juxtamembrane and the intracellular domains of the protein. B Heatmap showing the

of adherent cells expressing any of the variants (Fig. 2A). Interestingly, there is also a set of substrates that seem to be repulsive for mutant cells, given that lower adherence is observed when compared with the wild-type.

Using linear discriminant analysis effect size (LEfSe) that couples statistical significance with biological constancy and effect size estimation, we verified that the combinations of Fibronectin + Vitronectin, Fibronectin + Collagen IV, Laminin + Collagen IV, Fibronectin + Laminin, and Fibronectin + Collagen VI promote adherence of mutant cells independently of the variant expressed (Fig. 2B). Remarkably, Collagen VI or Vitronectin on their own, as well as the mixture of Fibronectin + Laminin + Collagen IV led to a reduction of cellmatrix adhesion in all mutants but not in wild-type cells. In accordance, scarce wild-type cells adhere to spots of Fibronectin + Vitronectin or Fibronectin + Collagen IV, whereas a higher number of mutant cells adhere to these ECM enriched sites (Fig. 2C). In contrast, Collagen VI and Vitronectin are attractive substrates for wild-type expressing cells but fewer cells are able to attach in the case of E-cadherin dysfunction. Altogether, this bias on ECM preference suggests that E-cadherin dysfunction activates a specific mechano-transduction program to regulate matrix adhesion. quantification of the adhesive abilities of AGS cells expressing the wild-type protein or the different variants. C Overview of matrix similarity analysis based on Pearson correlation data. Scaled distance (D) and Pearson correlation (E) between the adhesive profile of wild-type and E-cadherin mutant cells

\section{E-cadherin mutant cells exert increased traction forces in attractive ECM compositions}

It is well established that mechanical forces influence cellular and subcellular functions, including cell-matrix adhesion [27]. Therefore, we have evaluated the impact of ECM in cell mechanical loads through micropattern traction force microscopy. Traction measurements were conducted on polyacrylamide gels micropatterned with those ECM compositions inducing the most striking differences between functional and dysfunctional E-cadherin contexts. We selected the Fibronectin + Vitronectin combination to test an attractive condition, and Collagen VI to assess a repulsive situation for E-cadherin mutant cells (Fig. 3A and D).

Corroborating the previous results, cells expressing mutant E-cadherin display significant higher traction forces on gels patterned with Fibronectin + Vitronectin, when compared with cells transfected with the wild-type protein (Fig. 3B-C). In this substrate, the A634V mutant shows a mean sum of traction force of $28.0 \mathrm{nN}$, while the sum of all forces applied by cells expressing the wild-type version is $20.3 \mathrm{nN}(P=0.0171)$. Interestingly, AGS gastric cancer cells which are negative for E-cadherin expression present lower traction values than the wild-type model (18.0nN in Mock cells), further suggesting that cell-matrix 
A
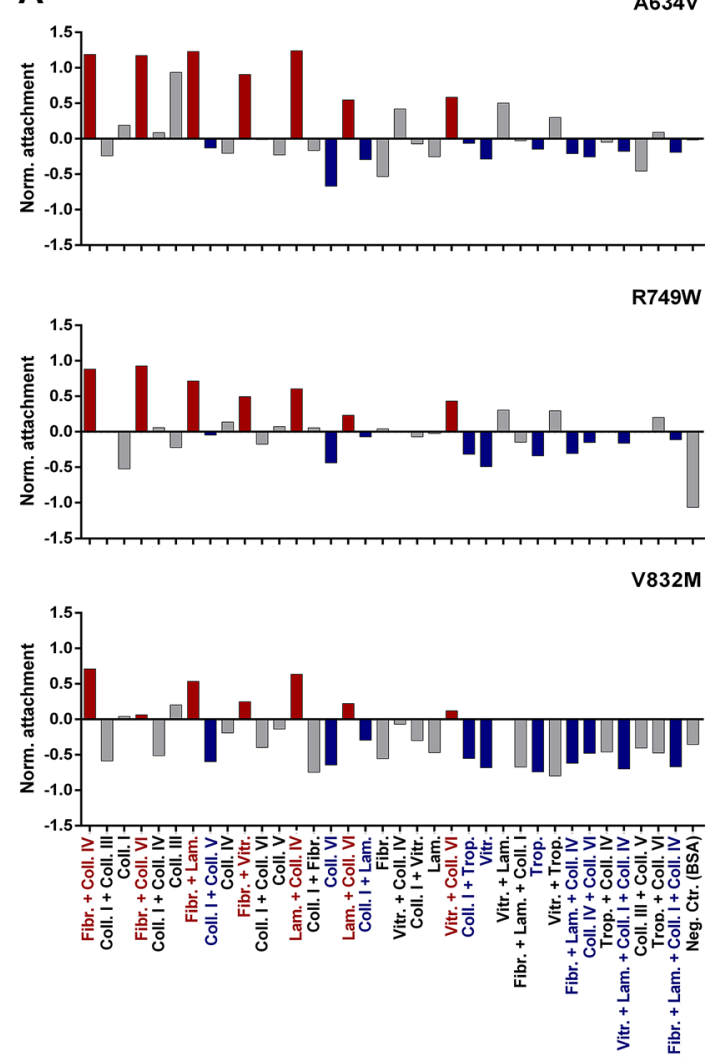

B

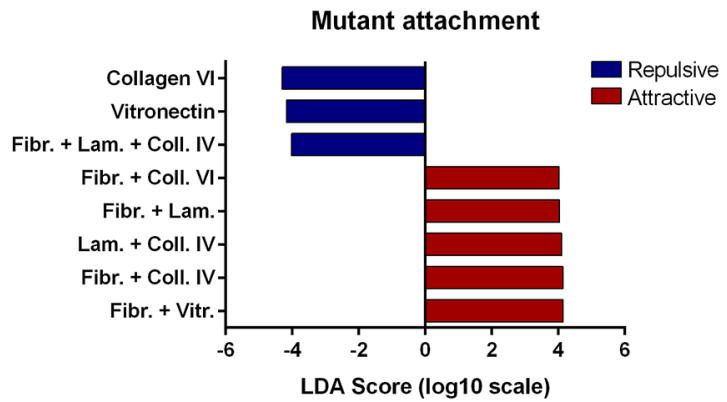

C

WT

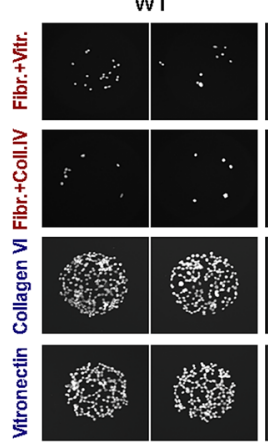

A634V

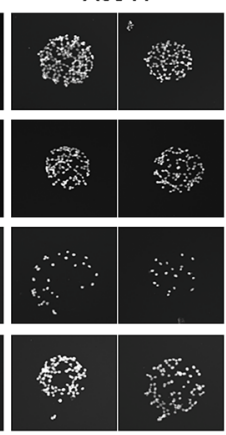

R749W

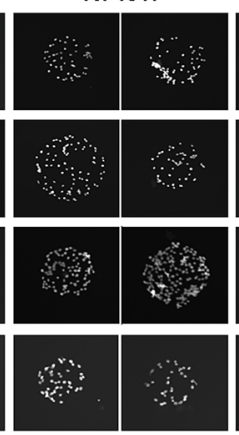

V832M

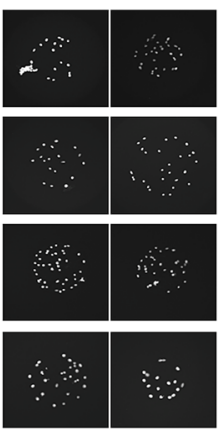

Fig. 2 Adhesion profile of A634V, R749W and V832M E-cadherin mutants. A The number of attached cells was quantified and normalized for cells expressing the wild-type protein. ECM compositions consistently inducing increased adhesive abilities are depicted in red whereas repulsive substrates are presented in blue. B LEfSe analy- sis (linear discriminant analysis effect size) displaying ECM compositions that induce significant differences in matrix attachment of mutants. C Representative images of spots illustrating cell attachment in the most mutant-attractive and -repulsive ECM combinations traction forces depend on E-cadherin expression and function. Cells exhibit the opposite behaviour on top of Collagen VI, since cells expressing mutant E-cadherin present weaker traction forces than those expressing the wild-type version ( $24.3 \mathrm{nN}$ for expression of A643V and $29.4 \mathrm{nN}$ for expression of the wild-type, Fig. 3E-F). Of relevance, wild-type cells adhere efficiently to Collagen VI and a remarkable difference is detected in the magnitudes of the vectors generated on this ECM, when compared with those produced on top of Fibronectin + Vitronectin (WT cells: $29.4 \mathrm{nN}$ in Col VI vs $20.3 \mathrm{nN}$ in FN + VTN, respectively, $P=0.0007)$.

Overall, our results demonstrate that E-cadherin mutant cells adhere preferentially to substrates in which they can exert increased traction forces. In repulsive substrates, there is reduced cytoskeletal tension, as weaker forces are applied in micropatterned gels. This indicates that with a suitable matrix composition, mutant cells have an increased ability to engage ECM receptors to apply mechanical forces and spread into the surrounding environment.

\section{Integrin $\beta 1$ is a candidate ECM receptor involved in the abnormal cell-matrix attachment of E-cadherin mutant cells}

Given that integrins are the main regulators of the cell-ECM crosstalk, we focused on the identification of those activated in E-cadherin dysfunctional cells. We implemented an ECM-integrin-guided method integrating the results of the ECM microarray profiling (Fig. 1) and a consensus view on the best-validated integrin ligands (Supplementary Table 2) $[17,18]$. This method considers the ability of integrins to mediate ECM-adhesion rather than gene/protein expression features, which are usually not correlated with functional effects. Among the integrins described as interactors of the ECM compositions included in the microarray, Integrin $\beta 1$ is the strongest candidate (Fig. 4A-B). Quantification of 
A

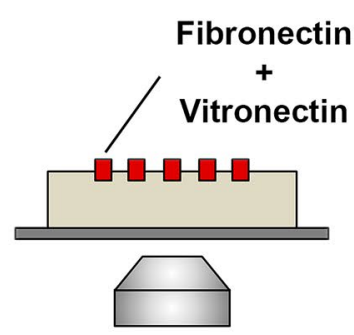

D

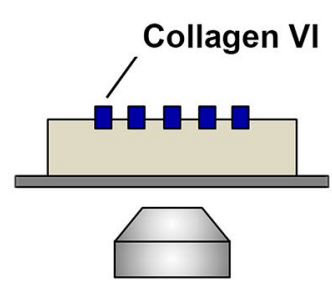

B

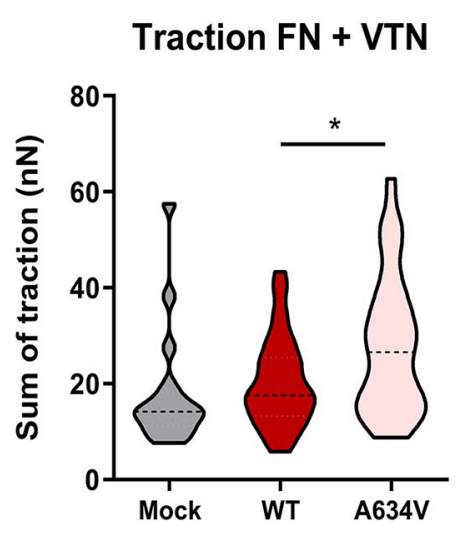

$\mathbf{E}$

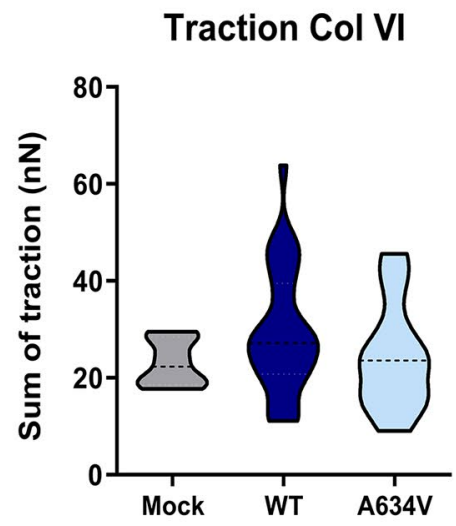

C
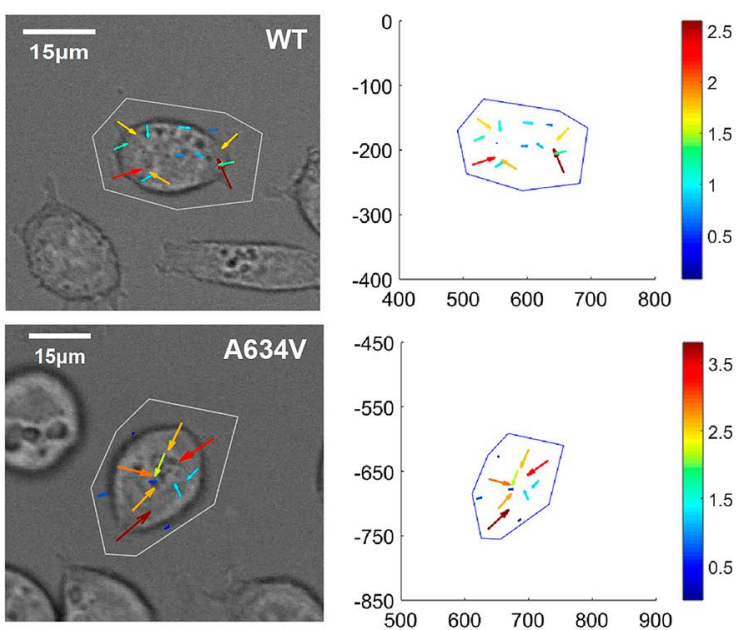

$\mathbf{F}$
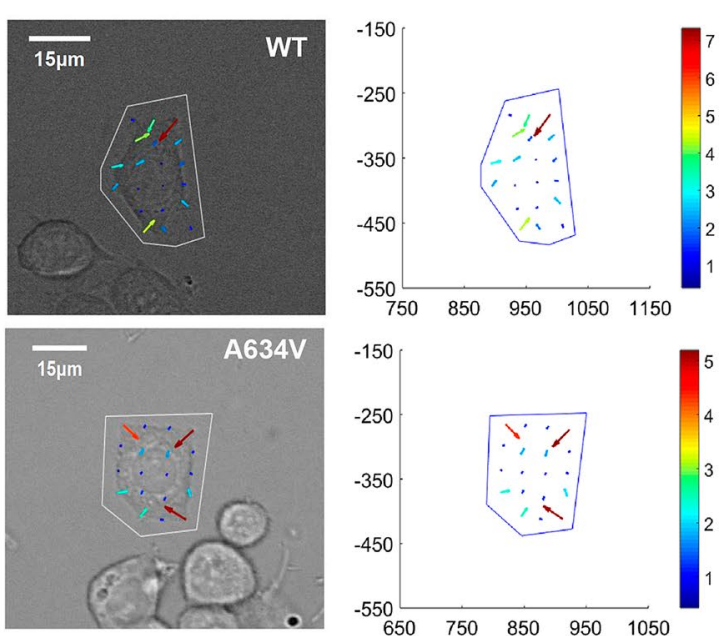

Fig. 3 Traction force of E-cadherin mutant cells cultured on different ECM compositions. Scheme illustrating traction force microscopy on polyacrylamide gels micropatterned with Fibronectin+Vitronectin (A) or Collagen VI (D). Quantification of traction forces exerted by negative, wild-type or mutant E-cadherin cells cultured on top

predicted molecular interactions occurring between receptors and ECM compositions inducing significant adhesion changes (identified by LEfSe analysis, Fig. 2B) pinpoints Integrin $\beta 1$ as the receptor mediating $67.9 \%$ of the interactions (72/106). In contrast to the $\beta$ subunit, there is no particular enrichment for the $\alpha$ subunit involved in ligandbinding of the 36 ECMs tested (Fig. 4C).

\section{Loss of Integrin $\beta 1$ function rescues misexpression of human E-cadherin in vivo}

To explore the importance of Integrin $\beta 1$ in the context of E-cadherin dysfunction, we established an in vivo model
Fibronectin+Vitronectin (B) or Collagen VI (E). C, F Differential interference contrast (DIC) images of cells overlapped with the corresponding traction force vectors are shown on the left. Traction force vectors alone are displayed on the right panels and colored according to magnitude $(\mathrm{nN})$

in Drosophila melanogaster. We first monitored the impact of overexpressing hE-cadherin in border cell migration across the Drosophila germline (Fig. 5A) [28]. Expression of wild-type hE-cad disrupts border cell migration towards the oocyte ( $\sim 49 \%$ of the expected distance, Fig. 5B-C), whereas border cells expressing the $\mathrm{R} 749 \mathrm{~W}$ mutant migrate similarly to cells expressing an inert UAS-driven transgene (UAS-mCherry).

We next misexpressed human E-cadherin in the developing Drosophila eye to investigate the genetic interaction with Integrin $\beta 1$. For that purpose, we engineered transgenic flies expressing WT or the R749W protein concomitantly with RNAi targeting mys/ßPS integrin, the Drosophila ortholog 

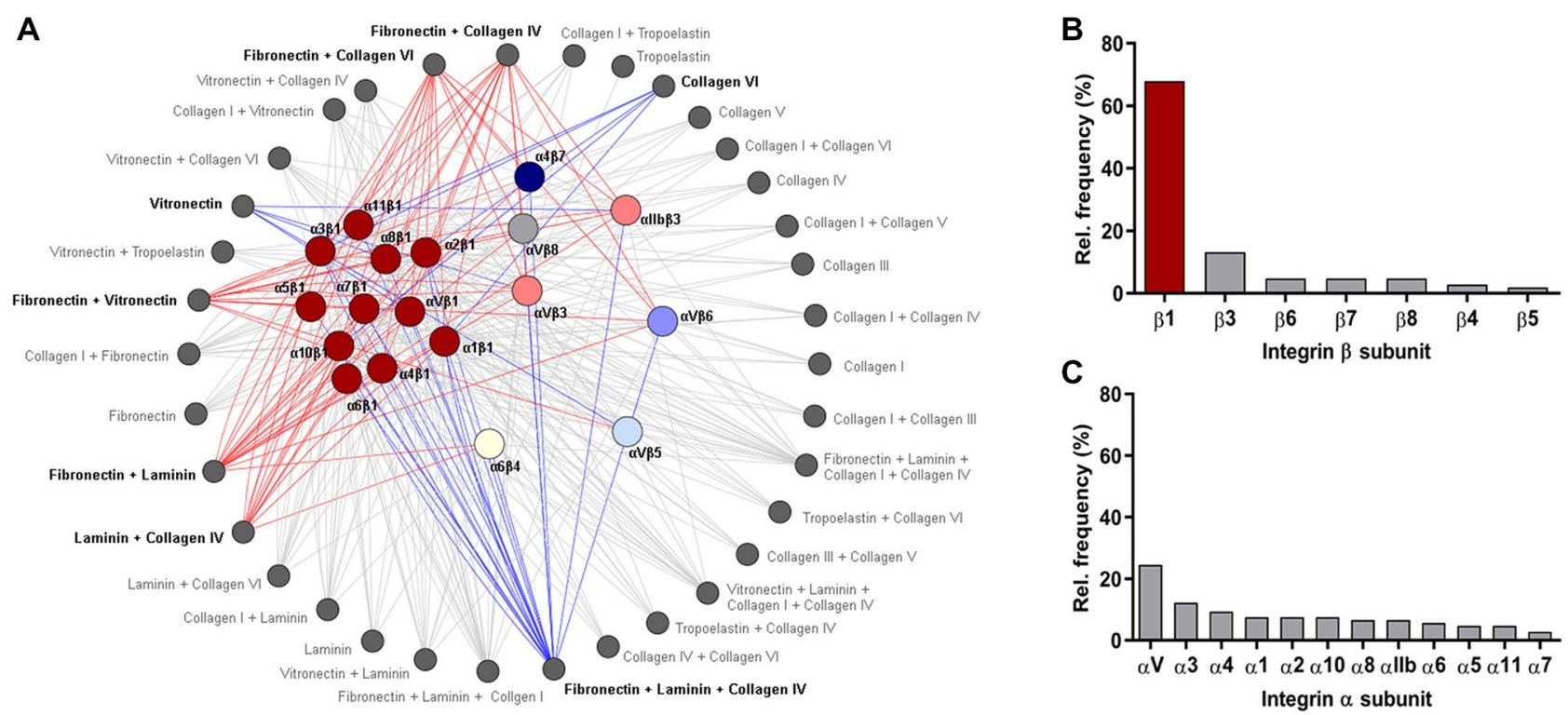

Fig. 4 ECM receptors involved in cell-matrix attachment of E-cadherin mutant cells. A A network diagram illustrating possible receptors of ECM components present in the microarray was constructed using Cytoscape. ECM-interactors were collected from published data. Colored nodes indicate ECM receptors and dark grey nodes represent the $36 \mathrm{ECM}$ compositions. Red edges indicate interactions

of Integrin $\beta 1$ (https://flybase.org/reports/FBgn0004657. $\mathrm{html})$. In contrast to flies overexpressing UAS-mCherry alone (control), the majority of flies expressing wild-type E-cadherin lose the ordered and symmetric structure of the eye, and instead show a "rough eye" phenotype frequently accompanied by necrosis and pigment loss (40\%, $n=174 / 431$, Fig. 5D-E). Expression of R749W further increases the penetrance of the most severe phenotype with the majority of flies presenting a rough eye with extensive necrotic areas and pigment loss $(66 \%, n=218 / 332)$. Strikingly, silencing $\beta$ PS integrin by RNAi rescues the most dramatic defects for both WT and R749W E-cadherin. Specifically, only $13 \%$ of the flies co-expressing UAS-hE-cad WT and UAS-mCherry RNAi show mild phenotypes (no phenotype or rough eye), whereas these mild phenotypes are observed in $40 \%$ and $69 \%$ of flies co-expressing UAS-hEcad WT with UAS-mysRNAi \#1 or with UAS-mysRNAi \#2, respectively. Likewise, the less severe phenotypes represent $26 \%$ of the cases when UAS-hE-cad R749W is co-expressed with mCherry RNAi, and increase their frequency to $39 \%$ and $41 \%$ when UAS-mysRNAi \#1 or UAS-mysRNAi \#2 are respectively co-expressed (Supplementary Fig. 1). Taken together, the genetic interactions detected in vivo reflect a role of Integrin $\beta 1$ in cellular defects elicited by E-cadherin dysfunction. of compositions that induce significant increased adhesion, and blue edges show connections of significant repulsive substrates. The interactions of ECM compositions that do not induce significant changes in cell adhesion are displayed in light grey. B, C Relative frequency of predicted interactions between integrin subunits $(\beta$ and $\alpha)$ and the tested ECM compositions

\section{Integrin $\beta 1$ silencing impairs invasion and increases cell-cell compaction of E-cadherin mutant cells}

To study the functional relevance of Integrin $\beta 1$ in human gastric cancer cells, we have performed its inhibition in AGS cells expressing either the wild-type or mutant forms of E-cadherin (Fig. 6A and Supplementary Fig. 2). Integrin $\beta 4$ was also inhibited to control for specificity of cellular effects induced by knockdown of Integrin $\beta 1$. Interestingly, cells expressing mutant forms of E-cadherin display significant increased levels of Integrin $\beta 1$ when compared to those of the wild-type, specifically: 1.76 fold in the $\mathrm{A} 634 \mathrm{~V}$ variant $(P=0.0076), 2.22$ fold in the R749W $(P=0.0011)$, and 1.54 fold in the V832M $(P=0.017)$. A small increase in Integrin $\beta 1$ was also observed upon inhibition of Integrin $\beta 4$, both in functional and dysfunctional E-cadherin cell lines (Supplementary Fig. 2).

The effect of integrin modulation on the invasive potential of cells was evaluated using matrigel invasion chambers. We observed that A634V, R749W and V832M induce an increase in the number of invasive cells, when compared with the wild-type protein $(2.23,2.15$ and 8.39 fold, respectively). Nevertheless, RNAi for Integrin $\beta 1$ rescues this phenotype, leading to a substantial decrease in invasion of the three E-cadherin mutants (Fig. 6B). In contrast, depletion 
A

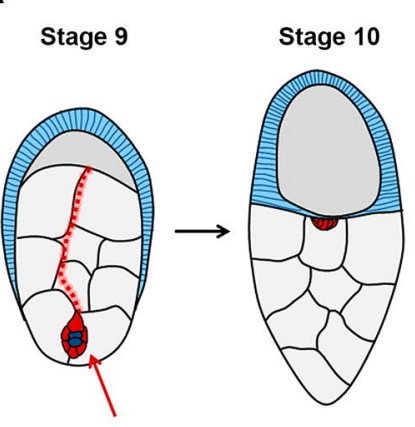

Border cell cluster expressing WT or R749W CDH1

\section{B Control}

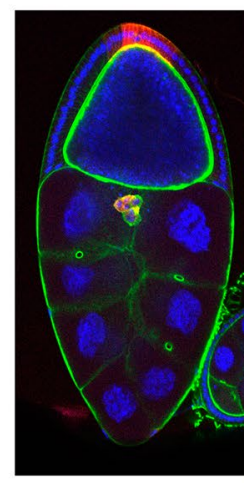

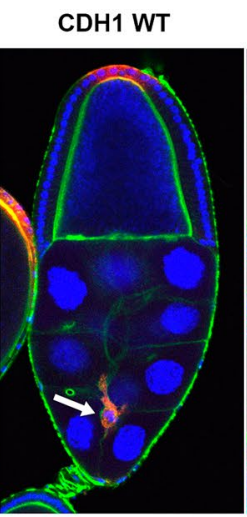

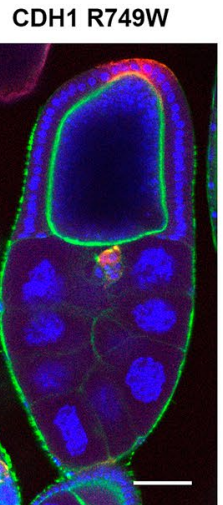

C Border cell migration

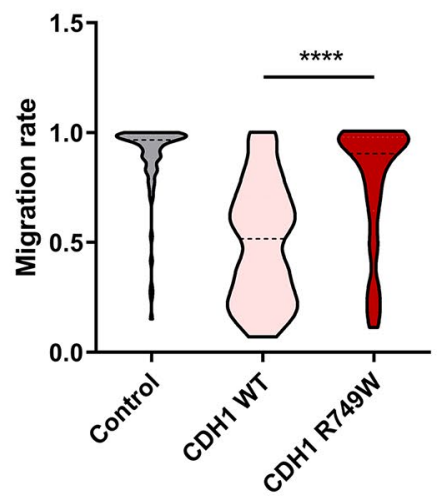

D

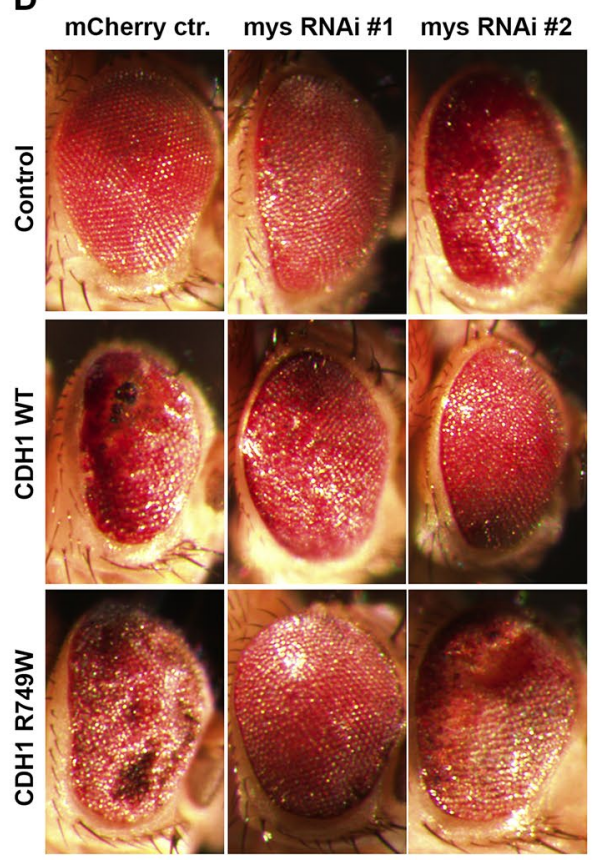

E

Eye phenotypes

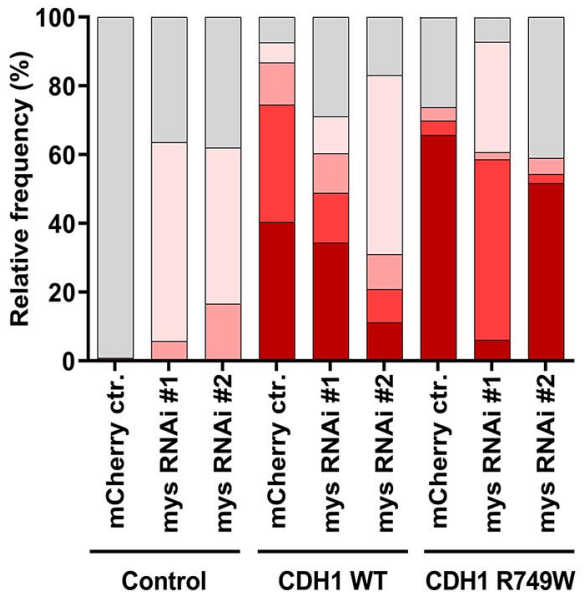

Rough + Necrosis + Pigm. Loss

Rough + Necrosis

$\square$ Rough + Pigm. Loss

$\square$ Rough

$\square$ No phenotype

Fig. 5 Genetic interaction between human E-cadherin and Integrin $\beta 1$ in Drosophila. A Schematic representation of border cell migration in stages 9 and 10 of oogenesis. B Migration phenotypes in stage 10 egg chambers. Border cells are marked in red by expression of UAS driven mCherry (red), phalloidin (green) stains F-actin and the nuclei are counterstained with DAPI (blue). Scale bar $=50 \mu \mathrm{m}$. C Quantitative analysis of the border cell migration index in cells expressing
CDH1 WT $(n=68)$ and $C D H 1$ R749W $(n=71)$ with the slbo-GAL4 driver, as well as in the control $(n=73)$. D Representative images of eye phenotypes from flies expressing the indicated UAS-driven transgenes in the developing eye with the GMR-Gal4 driver. E Graph indicates the relative frequency of fly phenotypes. For each condition, $n \geq 200$ flies analysed

by cells in closer proximity (Fig. 6C-F). Integrin $\beta 4$ seems to play an opposite role, with cells being further away from each other and generating bigger triangles upon its inhibition. This set of experiments suggests that Integrin $\beta 1$ is crucial for the initial steps of the invasive process mediated by E-cadherin dysfunction, possibly by promoting cell-matrix adhesion, cell-cell loosening and invasion of E-cadherin mutant cells. 


\section{Loss of E-cadherin and increased Integrin $\beta 1$ expression associate with tumour grade and patient overall survival}

Lastly, we investigated the E-cadherin/Integrin $\beta 1$ interplay using transcriptomic data of 262 gastric carcinoma samples, retrieved from TCGA [22]. By applying a threshold of 1.5fold change (0.58 in $\log 2)$ to the $\log 2$ ratio ITGB $1 / C D H 1$, we were able to discriminate two different groups of gastric carcinoma cases (Fig. 7A-B). Groups were defined as high $I T G B 1 /$ low $C D H 1$ expression (group $1, n=80$ ) and low $I T G B 1 /$ high $C D H 1$ expression (group $2, n=69$, Fig. 7C-D). Among cases included in these groups, an inverse correlation was detected between $I T G B I$ and $C D H 1$ expression (Pearson correlation $=-0.57, P<0.0001$, Fig. 7E). Cases displaying high $I T G B 1 /$ low $C D H 1$ expression were strongly associated with diffuse type gastric cancer and increased tumour grade (Fig. 7F-G). Moreover, these patients exhibited significantly lower overall survival, when compared with those harbouring tumours with a low ITGB1/high $C D H 1$ molecular phenotype (Fig. 7H). Additionally, using gastric cancer cell lines and available information concerning $\mathrm{CDH} 1$ mutation, DNA methylation, as well as microRNA expression, we observed that the ITGBI/CDHI inverse relationship is independent of the mechanism leading to E-cadherin inactivation (Supplementary Figs. 3 and 4). These results validate the relevance of the E-cadherin/Integrin $\beta 1$ pathway at the clinical level and highlight its potential as a biomarker of patient outcome.

\section{Discussion}

In the present work, our aim was to unravel the mechanisms and key players underlying the invasive process mediated by E-cadherin mutations. Evidence from previous reports demonstrates that mechanoregulation plays an important role in epithelial tissue homeostasis, and implicates the interdependence between cadherins and integrins [13, 29, 30]. Cadherins are fundamental for intercellular junctional stability, whereas integrins are the main receptors for components of the ECM $[10,12]$. Taking this into account, we postulated that E-cadherin mutations cause an imbalance at the cell-ECM interface with critical impact in cancer cell invasion.

Herein, we have addressed our aim through a novel ECM microarray approach, which is based upon cell functional activity, in contrast to gene expression or transcriptome analysis that only consider differential mRNA levels [31]. Using this platform, we have studied the (cell-ECM) adhesive function of cells expressing distinct E-cadherin variants associated with HDGC and compared it with that of the wild-type protein. We verified that different variants confer different adhesion profiles, in accordance with our previous work reporting that each E-cadherin mutation dictates a specific cell behaviour [14, 32]. Despite the marked difference between adhesion profiles, we verified that the most mutantattractive compositions combine Fibronectin and structural ECM molecules such as Collagen IV, Collagen VI or Laminin. Fibronectin has been described to work as a bridge between structural components of the basement membrane, serving as a substrate for integrin engagement and clustering in many cell types [33-35]. Differently, Collagen IV fibers are major components within the basement membrane, and form a scaffold for association of other proteins [33, 36]. Data available concerning Vitronectin are still scarce, but it has been postulated that this adhesive glycoprotein plays a role in the provisional matrix of tumours, promoting cell adhesion and matrix degradation by binding to integrins, plasminogen activator inhibitor-1 (PAI-1) and urokinase plasminogen activator receptor (UPAR) [37]. Given the complexity of the basement membrane and the unique functions of the different ECM proteins, it is expected that cells with malignant potential do not adhere to matrices of a single component such as Collagen VI or Vitronectin. We may speculate that, under these conditions, mutant cells are able to survive in an anchorage independent manner.

It is well known that the biophysical properties of the ECM regulate tensional homeostasis [9]. We have therefore measured traction forces exerted by wild-type and mutant cells on attractive and repulsive ECM substrates. Higher traction forces were detected in matrices to which cells adhere more efficiently, and while mutant cells exert increased tension on Fibronectin + Vitronectin, a lower mechanical input is observed in Collagen VI. The opposite effect was seen in wild-type cells. Of note, Fibronectin and Vitronectin are ligands for RGD-binding integrins (integrins with affinity for Arg-Gly-Asp sequence), whereas the receptors for collagen belong to a distinct integrin class, named $\alpha \mathrm{A}$-domain-containing integrins [17]. This suggests that an integrin switch may occur from a competent to an incompetent E-cadherin context, involving activation of the most promiscuous class of integrins.

By combining ECM adhesion profiles and available data on specific ECM receptors, we identified Integrin $\beta 1$ as the receptor most likely involved in the response of E-cadherin dysfunctional cells. Integrin $\beta 1$ overexpression was already described in gastric cancer and associated with vascular invasion and lymph node metastasis $[38,39]$. The $\beta 1$ 
A

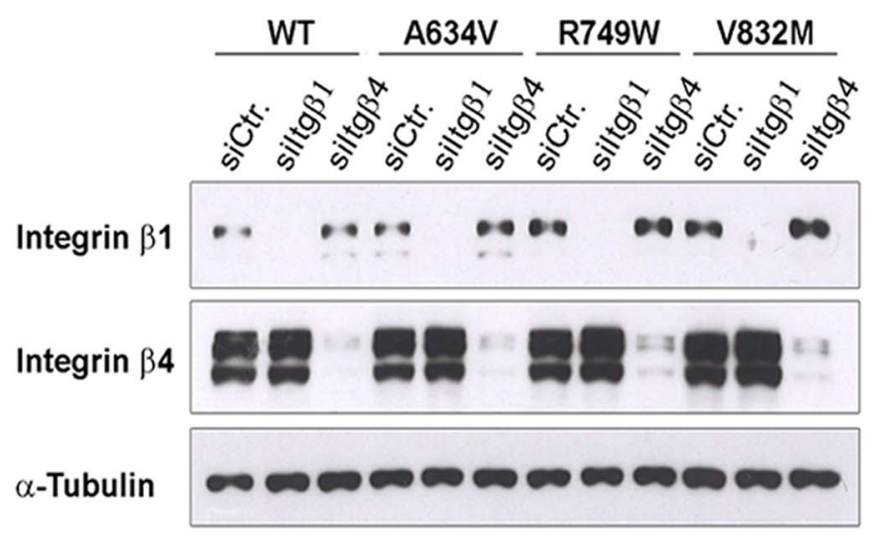

B

Invasion

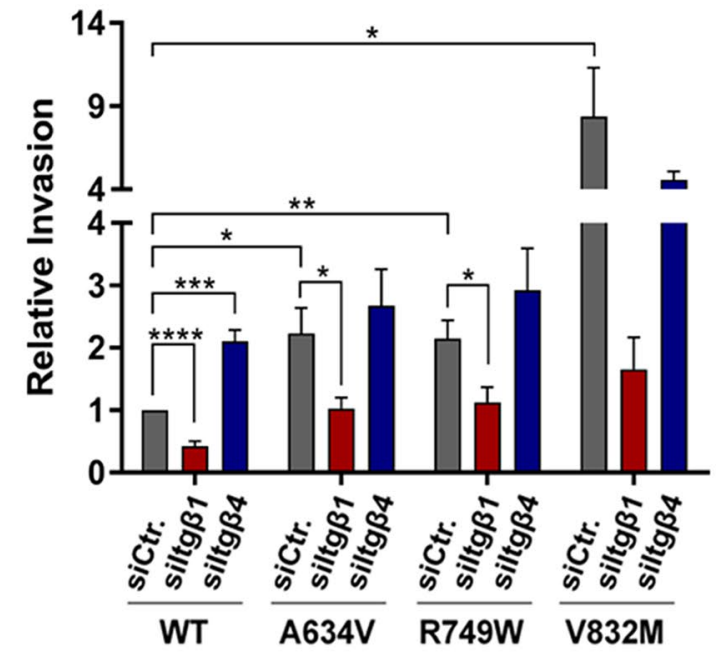

C
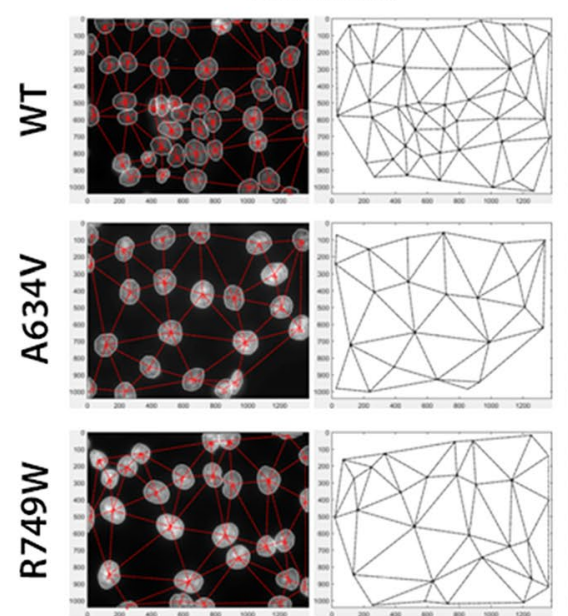

siltg $\beta 1$
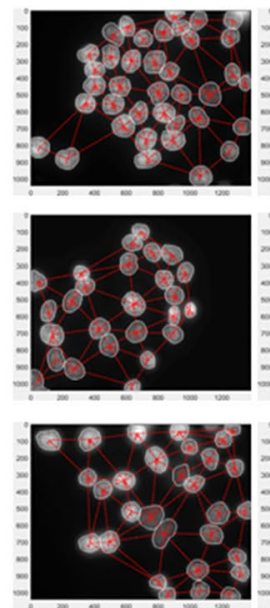

Area

E
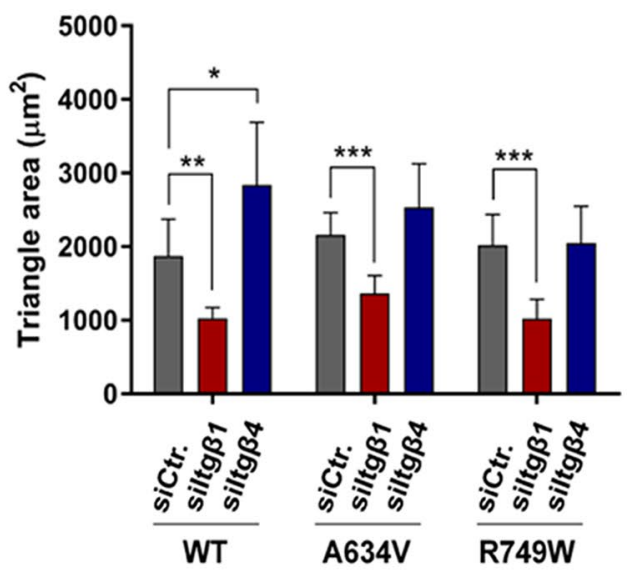

F
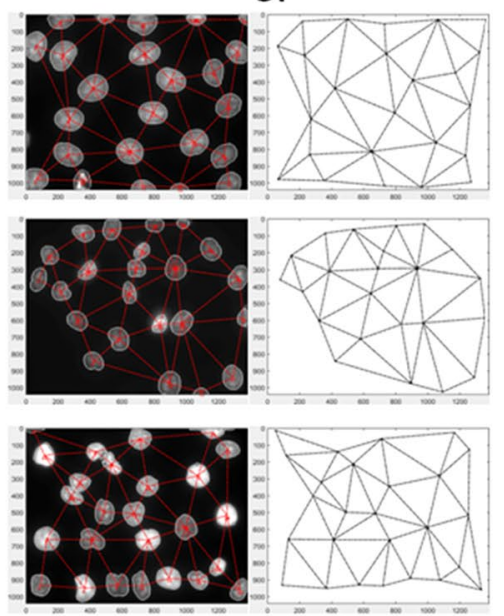

Internuclear distance

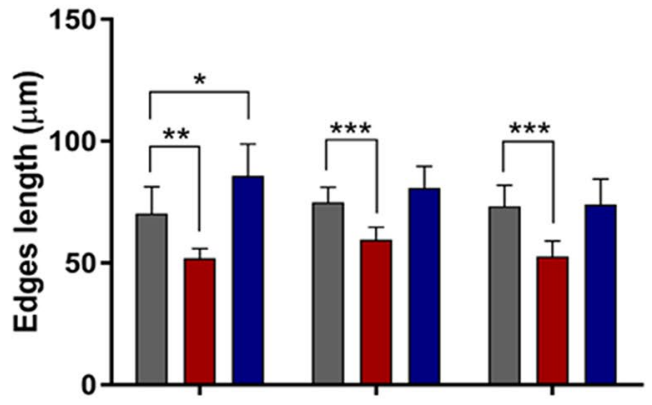

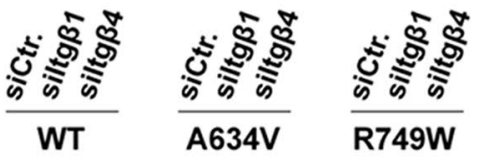


4Fig. 6 Integrin $\beta 1$ functional significance in an E-cadherin mutant setting. Specific inhibition of Integrin $\beta 1$ or $\beta 4$ by siRNA was performed in cells stably transfected with wild-type or mutant E-cadherin. A Integrin $\beta 1$ and Integrin $\beta 4$ levels were analysed by Western Blot. $\alpha$-Tubulin was used as a loading control. B Cell invasive ability upon integrin modulation was evaluated through matrigel invasion chambers. C Cellular distribution patterns elicited by integrin inhibition. Cell nuclei overlapped with the corresponding network (on the left) and final networks (on the right) are presented. D Scheme illustrating the measurement of cell-cell connection and cell distribution patterns through automated assembly of neighbouring nuclei. Quantitative analysis of networks regarding triangle areas $(\mathbf{E})$ and edges length $(\mathbf{F})$

subunit was found to be crucial for peritoneal dissemination of human gastric carcinoma, both in patient samples and xenograft models [40-42]. Nevertheless, a causal relationship between E-cadherin loss of function and Integrin $\beta 1$ activity remained elusive.

A possible E-cadherin/Integrin $\beta 1$ linkage was addressed in vivo by the establishment of transgenic fly lines expressing wild-type and mutant human E-cadherin, in which we modulated the expression of the Drosophila ortholog of Integrin $\beta 1$ ( $\beta \mathrm{PS})$. Our experiments demonstrated that integrin expression contributes to the severe effects of human E-cadherin misexpression, since depletion of $\beta$ PS integrin reduces the prevalence of the stronger phenotypic defects in eye morphology. We then elucidated the biological significance of Integrin $\beta 1$ in E-cadherin defective cells by taking advantage of our in vitro cancer cell model. We found that cells expressing E-cadherin missense variants indeed exhibit increased levels of Integrin $\beta 1$. Remarkably, Integrin $\beta 1$ downregulation increased cell-cell compaction and induced a marked decrease in the invasive ability of mutant cells. These results corroborate data showing that Integrin $\beta 1$ expression induces scattering of mouse epithelial cells through regulation of the cadherin-catenin complex, as well as remodelling of the actin cytoskeleton [43]. In addition, it is known that Integrin $\beta 1$ inhibition blocks E-cadherin internalization, leading to increased intercellular adhesion [30].

Finally, we found an inverse correlation between E-cadherin and Integrin $\beta 1$ expression in a series of 262 gastric carcinoma cases, confirming that E-cadherin loss triggers deregulation of cell-matrix interactions regardless of the mechanism leading to $\mathrm{CDH} 1$ inactivation. Cases presenting low E-cadherin expression levels exhibited high levels of integrin and were associated with increased tumour grade and poor patient overall survival. In agreement with the present findings, our group has previously reported overexpression of Laminin $\gamma 2$ and decreased E-cadherin in gastric cancer cell lines, Drosophila models and primary tumours [44]. Laminin $\gamma 2$ - a major basement membrane componentallows E-cadherin defective cells to survive and invade, contributing to gastric carcinogenesis [44]. Importantly, $\beta 1$ integrins $(\alpha 3, \alpha 6$ and $\alpha 7)$ are receptors for laminins.

A critical issue for future research should cover the characterization of the ECM proteome and its regulators, or "matrisome", in normal, pre-malignant and malignant gastric epithelia, which remain largely unexplored and would complement our understanding of the oncogenic cell-ECM affair.

\section{Conclusion}

This work shed light on the molecular machinery implicated in the invasive process mediated by E-cadherin mutations. We provided evidence that E-cadherin dysfunctional cells activate specific integrins, namely Integrin $\beta 1$, for efficient adhesion and dissemination through the ECM. We propose that Integrin $\beta 1$ can be used in the clinics as a new predictive biomarker of gastric cancer progression. 


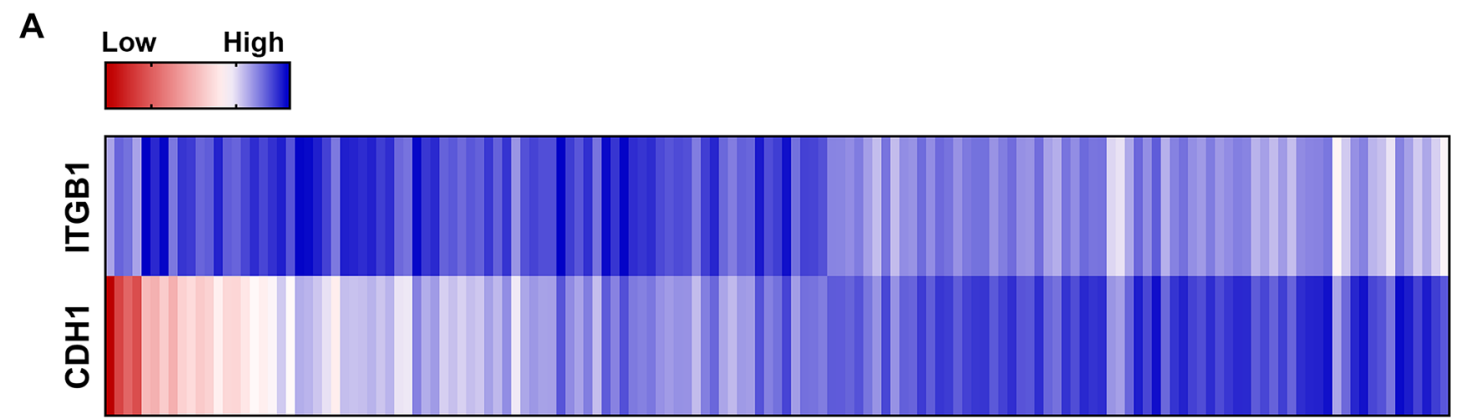

B

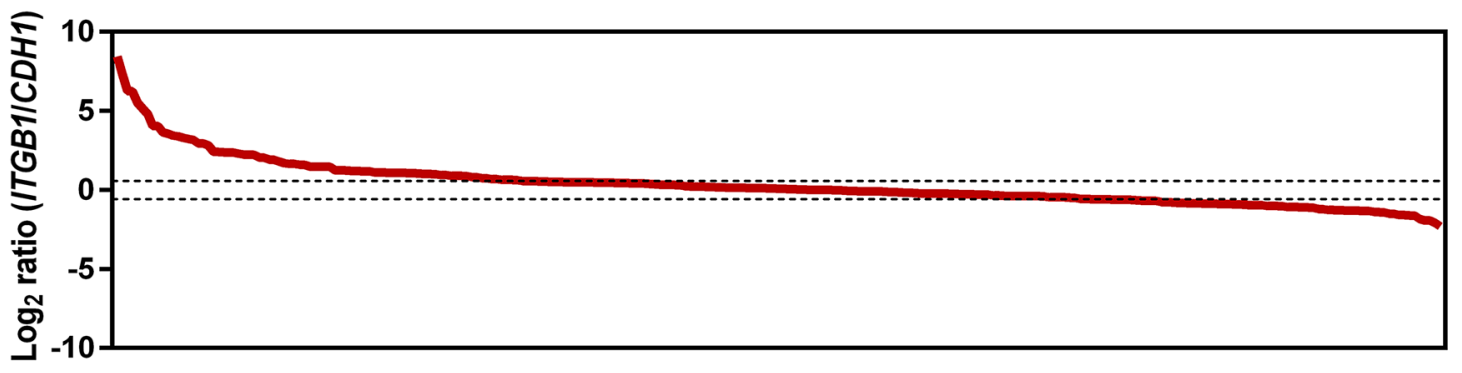

C

Group I

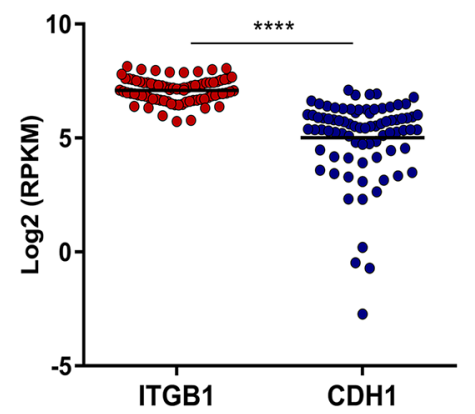

$\mathbf{F}$

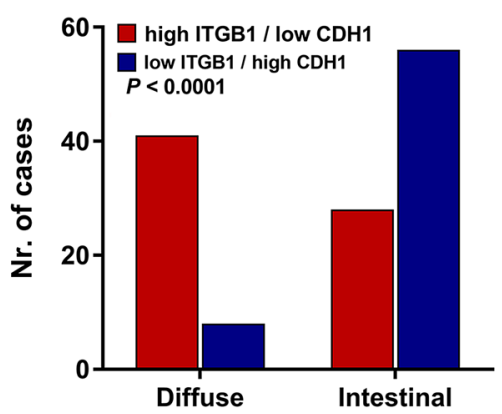

D

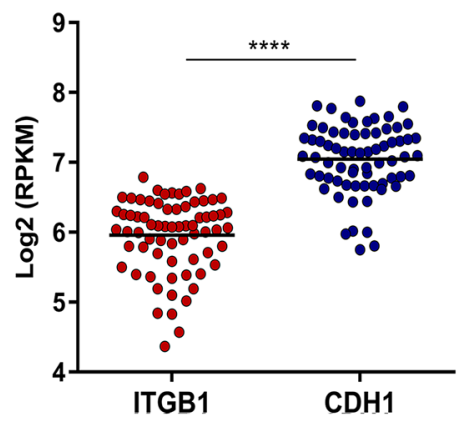

G

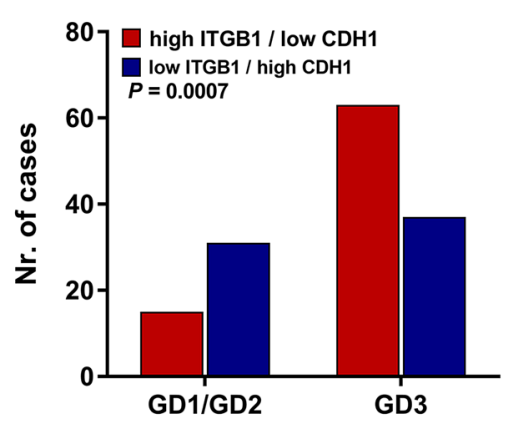

E

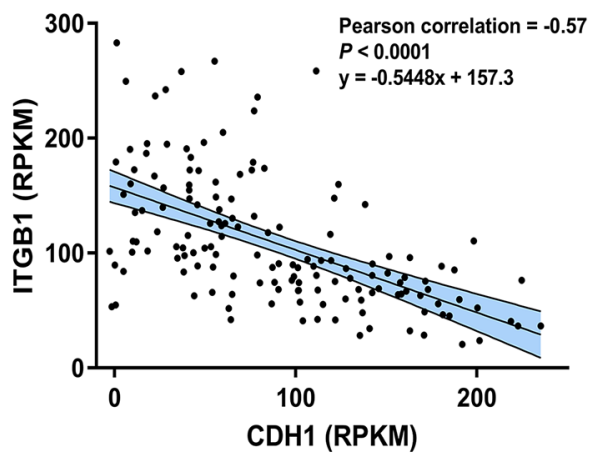

H

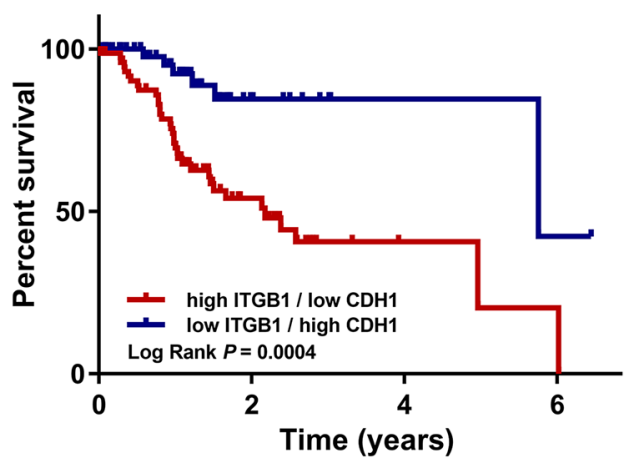

Fig. 7 Clinical relevance of the Integrin $\beta 1 /$ E-cadherin interplay in human gastric carcinoma samples. A Heatmap representing ITGBI and $C D H 1$ mRNA levels in 262 gastric cancer samples according to ITGB1/CDH1 $\log 2$ ratio. B Graph of $\log 2$ ratio depicting two groups of samples following implementation of a 1.5-fold threshold: group I displaying high $I T G B 1 / C D H 1$ ratio and group II low $I T G B 1 / C D H 1$ ratio. C, D Differential expression of ITGBI and $C D H 1$ in the two patient groups. E Pearson correlation of ITGB1 and $C D H 1$ reads per kilobase of transcript per million mapped reads (RPKM). Association between $I T G B 1$ and $C D H 1$ levels with tumour histological type $(\mathbf{F})$ and grade $(\mathbf{G})$. H Survival plot illustrating overall survival of patients exhibiting either high $I T G B 1 /$ low $C D H 1$ or low $I T G B 1 /$ high $C D H 1$ expression 
Supplementary Information The online version contains supplementary material available at https://doi.org/10.1007/s10120-021-01239-9.

Acknowledgements The authors acknowledge the support of the BioSciences Screening (BS) and Advanced Light Microscopy (ALM) i3S Scientific Platforms, which are members of the national PPBI-Portuguese Platform of Bioimaging infrastructure (PPBI-POCI-01-0145-FEDER-022122).

Author contributions Study concept and design: JF, EMS and RS. Data acquisition: JF, RMF, HX, MG, ABC, JC, AFM. Data analysis and interpretation: JF, RMF, PC, CF, MLS, DS, EMS and RS. Writing of the original draft: JF. Editing and critical review of the manuscript: RMF, HX, MG, ABC, JC, AFM, PC, CF, MLS, DS, EMS and RS. All authors approved the final version of manuscript.

Funding This work was financed by FEDER funds through the Operational Programme for Competitiveness Factors (COMPETE 2020), Programa Operacional de Competitividade e Internacionalização (POCI) and Programa Operacional Regional do Norte (Norte 2020); by National Funds through the Portuguese Foundation for Science and Technology (FCT projects PTDC/MED-GEN/30356/2017, PTDC/ BTM-SAL/30383/2017, PTDC/BIM-ONC/0281/2014, NORTE-010145-FEDER-000029, and doctoral grant SFRH/BD/130708/2017MG); and by the National Science Foundation (NSF grants CMMI1362922 and CMMI-1910401). RMF and EMS are funded by the "FCT Scientific Employment Stimulus-Individual Call" program (CEECIND/01854/2017 and CEECIND/00622/2017). We acknowledge the American Association of Patients with Hereditary Gastric Cancer "No Stomach for Cancer" for funding Seruca's and Figueiredo's research.

\section{Declarations}

Conflict of Interest The authors declare no competing of financial and non-financial interest.

Open Access This article is licensed under a Creative Commons Attribution 4.0 International License, which permits use, sharing, adaptation, distribution and reproduction in any medium or format, as long as you give appropriate credit to the original author(s) and the source, provide a link to the Creative Commons licence, and indicate if changes were made. The images or other third party material in this article are included in the article's Creative Commons licence, unless indicated otherwise in a credit line to the material. If material is not included in the article's Creative Commons licence and your intended use is not permitted by statutory regulation or exceeds the permitted use, you will need to obtain permission directly from the copyright holder. To view a copy of this licence, visit http://creativecommons.org/licenses/by/4.0/.

\section{References}

1. Lecuit T, Yap AS. E-cadherin junctions as active mechanical integrators in tissue dynamics. Nat Cell Biol. 2015;17:533-9.

2. Paredes J, Figueiredo J, Albergaria A, et al. Epithelial E- and P-cadherins: role and clinical significance in cancer. Biochim Biophys Acta. 2012;1826:297-311.

3. Guilford P, Hopkins J, Harraway J, et al. E-cadherin germline mutations in familial gastric cancer. Nature. 1998;392:402-5.

4. Blair VR, McLeod M, Carneiro F, et al. Hereditary diffuse gastric cancer: updated clinical practice guidelines. Lancet Oncol. 2020;21:e386-97.
5. Hansford S, Kaurah P, Li-Chang H, et al. Hereditary diffuse gastric cancer syndrome: cdh1 mutations and beyond. JAMA Oncol. 2015;1:23-32.

6. Oliveira C, Pinheiro H, Figueiredo J, Seruca R, Carneiro F. Familial gastric cancer: genetic susceptibility, pathology, and implications for management. Lancet Oncol. 2015;16:e60-70.

7. Carneiro F, Huntsman DG, Smyrk TC, et al. Model of the early development of diffuse gastric cancer in E-cadherin mutation carriers and its implications for patient screening. J Pathol. 2004;203:681-7.

8. Goodwin K, Lostchuck EE, Cramb KML, Zulueta-Coarasa T, Fernandez-Gonzalez R, Tanentzapf G. Cell-cell and cell-extracellular matrix adhesions cooperate to organize actomyosin networks and maintain force transmission during dorsal closure. Mol Biol Cell. 2017;28:1301-10.

9. DuFort CC, Paszek MJ, Weaver VM. Balancing forces: architectural control of mechanotransduction. Nat Rev Mol Cell Biol. 2011;12:308-19.

10. Rubsam M, Mertz AF, Kubo A, et al. E-cadherin integrates mechanotransduction and EGFR signaling to control junctional tissue polarization and tight junction positioning. Nat Commun. 2017;8:1250.

11. Hamidi H, Ivaska J. Every step of the way: integrins in cancer progression and metastasis. Nat Rev Cancer. 2018;18:533-48.

12. Cooper J, Giancotti FG. Integrin signaling in cancer: mechanotransduction, stemness, epithelial plasticity, and therapeutic resistance. Cancer Cell. 2019;35:347-67.

13. Canel M, Serrels A, Frame MC, Brunton VG. E-cadherinintegrin crosstalk in cancer invasion and metastasis. J Cell Sci. 2013;126:393-401.

14. Figueiredo J, Soderberg O, Simoes-Correia J, Grannas K, Suriano G, Seruca R. The importance of E-cadherin binding partners to evaluate the pathogenicity of E-cadherin missense mutations associated to HDGC. Eur J Hum Genet. 2013;21:301-9.

15. Berg S, Kutra D, Kroeger T, et al. ilastik: interactive machine learning for (bio) image analysis. Nat Methods. 2019;16:1226-32.

16. Carpenter AE, Jones TR, Lamprecht MR, et al. Cell Profiler: image analysis software for identifying and quantifying cell phenotypes. Genome Biol. 2006;7:R100.

17. Humphries JD, Byron A, Humphries MJ. Integrin ligands at a glance. J Cell Sci. 2006;119:3901-3.

18. Jin H, Varner J. Integrins: roles in cancer development and as treatment targets. Br J Cancer. 2004;90:561-5.

19. Shannon P, Markiel A, Ozier O, et al. Cytoscape: a software environment for integrated models of biomolecular interaction networks. Genome Res. 2003;13:2498-504.

20. Xu H, Donegan S, Dreher JM, et al. Focal adhesion displacement magnitude is a unifying feature of tensional homeostasis. Acta Biomater. 2020;113:372-9.

21. Mestre T, Figueiredo J, Ribeiro AS, Paredes J, Seruca R, Sanches JM. Quantification of topological features in cell meshes to explore E-cadherin dysfunction. Sci Rep. 2016;6:25101.

22. Cancer Genome Atlas Research Network. Comprehensive molecular characterization of gastric adenocarcinoma. Nature. 2014;513:202-9.

23. Suriano G, Oliveira C, Ferreira P, et al. Identification of CDH1 germline missense mutations associated with functional inactivation of the E-cadherin protein in young gastric cancer probands. Hum Mol Genet. 2003;12:575-82.

24. Kaurah P, MacMillan A, Boyd N, et al. Founder and recurrent CDH1 mutations in families with hereditary diffuse gastric cancer. JAMA. 2007;297:2360-72.

25. Simoes-Correia J, Figueiredo J, Oliveira C, et al. Endoplasmic reticulum quality control: a new mechanism of E-cadherin regulation and its implication in cancer. Hum Mol Genet. 2008; 17:3566-76. 
26. More H, Humar B, Weber W, et al. Identification of seven novel germline mutations in the human E-cadherin (CDH1) gene. Hum Mutat. 2007;28:203.

27. Broders-Bondon F, Nguyen Ho-Bouldoires TH, FernandezSanchez ME, Farge E. Mechanotransduction in tumor progression: the dark side of the force. J Cell Biol. 2018;217:1571-87.

28. Stuelten CH, Parent CA, Montell DJ. Cell motility in cancer invasion and metastasis: insights from simple model organisms. Nat Rev Cancer. 2018;18:296-312.

29. Weber GF, Bjerke MA, DeSimone DW. Integrins and cadherins join forces to form adhesive networks. J Cell Sci. 2011;124:1183-93.

30. Canel M, Serrels A, Miller D, et al. Quantitative in vivo imaging of the effects of inhibiting integrin signaling via Src and FAK on cancer cell movement: effects on E-cadherin dynamics. Cancer Res. 2010;70:9413-22.

31. Brafman DA, de Minicis S, Seki E, et al. Investigating the role of the extracellular environment in modulating hepatic stellate cell biology with arrayed combinatorial microenvironments. Integr Biol. 2009;1:513-24.

32. Figueiredo J, Melo S, Gamet K, et al. E-cadherin signal sequence disruption: a novel mechanism underlying hereditary cancer. Mol Cancer. 2018;17:112.

33. Mouw JK, Ou G, Weaver VM. Extracellular matrix assembly: a multiscale deconstruction. Nat Rev Mol Cell Biol. 2014;15:771-85.

34. Ruoslahti E. Fibronectin and its receptors. Annu Rev Biochem. 1988;57:375-413.

35. Choquet D, Felsenfeld DP, Sheetz MP. Extracellular matrix rigidity causes strengthening of integrin-cytoskeleton linkages. Cell. 1997;88:39-48.

36. Yurchenco PD, Patton BL. Developmental and pathogenic mechanisms of basement membrane assembly. Curr Pharm Des. 2009;15:1277-94.
37. Madsen CD, Ferraris GM, Andolfo A, Cunningham O, Sidenius N. uPAR-induced cell adhesion and migration: vitronectin provides the key. J Cell Biol. 2007;177:927-39.

38. Ren J, Xu S, Guo D, Zhang J, Liu S. Increased expression of alpha5beta1-integrin is a prognostic marker for patients with gastric cancer. Clin Transl Oncol. 2014;16:668-74.

39. Ke JJ, Shao QS, Ling ZQ. Expression of E-selectin, integrin beta1 and immunoglobulin superfamily member in human gastric carcinoma cells and its clinicopathologic significance. World J Gastroenterol. 2006;12:3609-11.

40. Kawamura T, Endo Y, Yonemura Y, et al. Significance of integrin alpha2/beta1 in peritoneal dissemination of a human gastric cancer xenograft model. Int J Oncol. 2001;18:809-15.

41. Matsuoka T, Yashiro M, Nishimura S, et al. Increased expression of alpha2beta1-integrin in the peritoneal dissemination of human gastric carcinoma. Int J Mol Med. 2000;5:21-5.

42. Fujita S, Suzuki H, Kinoshita M, Hirohashi S. Inhibition of cell attachment, invasion and metastasis of human carcinoma cells by anti-integrin beta 1 subunit antibody. Jpn J cancer Res. 1992;83:1317-26.

43. Gimond C, van der Flier A, van Delft S, et al. Induction of cell scattering by expression of beta1 integrins in beta1-deficient epithelial cells requires activation of members of the rho family of GTPases and downregulation of cadherin and catenin function. J Cell Biol. 1999;147:1325-40.

44. Caldeira J, Figueiredo J, Bras-Pereira C, et al. E-cadherin-defective gastric cancer cells depend on Laminin to survive and invade. Hum Mol Genet. 2015;24:5891-900.

Publisher's Note Springer Nature remains neutral with regard to jurisdictional claims in published maps and institutional affiliations. 